\title{
Hacia una elucidación del campo de estudios sobre cine en Chile
}

\section{Towards a Clarification of Film Studies Field in Chile}

\author{
Hans Stange \\ Instituto de la imagen, Universidad de Chile. Santiago \\ hstangemarcus@yahoo.es
}

\author{
Claudio Salinas \\ Instituto de la imagen, Universidad de Chile. Santiago \\ claudiorsm@yahoo.com
}

Resumen - El campo cultural constituido en torno al cine se articula alrededor de múltiples dimensiones: la producción fílmica, los mercados, las políticas estatales e institucionales, los públicos, los contextos culturales e históricos y el ámbito de los estudios y la teoría. Este último tiene en Chile, al parecer, un desarrollo fragmentario, desigual y no suficientemente asentado y legitimado. El presente artículo pretende ofrecer un panorama sobre los estudios de cine en Chile, centrándose en tres áreas relevantes (la institucionalidad, los modelos y problemas teóricos y las prácticas críticas) para enunciar, a partir de esta «revisión», por cierto parcial, un conjunto de preguntas respecto a la necesidad de consolidación de estos estudios, sus orientaciones futuras y sus posibilidades de diálogo con otros ámbitos del campo cinematográfico.

Palabras clave: cine en Chile, campo cultural, teoría del cine.

Abstract - The cultural field formed around cinema has been built in several dimensions: film production, markets, public policies, audiences, cultural and historical contexts and film studies and its theory. This one has had in Chile a fragmented, uneven and not sufficiently established nor legitimated development. This paper aims to provide an overview of film studies in Chile, focusing on three important areas (institutions, theories and problems and, by last, criticism) to state, from this partial «review», a set of questions regarding the need to consolidate these studies, their future and their ability to dialogue with other areas of Cinema.

Keywords: cinema in Chile, cultural theory, film studies. 


\section{INTRODUCCIÓN}

¿Cuál es el campo en el que se introduce quien comienza a investigar sobre cine en Chile? A poco andar, investigadores y tesistas se percatan de que la literatura no es abundante, que ciertas películas son a menudo de difícil acceso o, más aún, se conocen sólo por referencias; que las estadísticas están dispersas, son parciales o incompletas. En fin, pareciera ser que siempre se está por comenzar, que recién se está descubriendo al cine como un fértil campo de estudios.

En esta situación, son pocos y oscuros los marcos para el investigador. ¿Qué leer? ¿A cuáles tradiciones teóricas arrimarse? ¿Qué informes y catálogos consultar? ¿Cuáles son los autores de referencia, los temas y problemas recurrentes que han movilizado la investigación, los grandes debates, las corrientes y escuelas principales? A algunos les parecerá que se trata de un territorio vacío, inexistente: un páramo. A otros, más optimistas, les parecerá ciertamente un campo inarticulado y sin desarrollo, empero identificarían ciertos autores, tendencias y temáticas más o menos claras bajo las cuales cobijarse y emprender la tarea de la investigación. En cualquier caso, nadie estaría dispuesto a sostener que hoy por hoy existe un campo de estudios del cine consolidado en nuestro país.

¿Cuáles son las pistas y señales que nos podrían avisar de la existencia de un campo de estudios o, también, de su emergencia? ¿Qué cosas inquirimos de un determinado campo cultural? Porque los estudios sobre cine participan de un campo de producción cultural particular: el cine. Éste constituye uno de sus ámbitos junto a otros espacios fácilmente identificables: la producción cinematográfica, la distribución y el mercado cinematográfico, las audiencias, la crítica especializada, las instituciones formadoras y las políticas públicas y privadas de fomento, etc.

Afirmar que el cine constituye un campo cultural significa afirmar que es un entramado de relaciones de fuerzas entre diversos actores sociales, institucionales y culturales, al interior del cual se producen no sólo obras materiales (las películas) sino también discursos, interpretaciones y valores, tanto económicos como simbólicos. ${ }^{1}$ El carácter de un campo cultural depende de la disposición de estas relaciones, por lo que no podríamos aventurar aquí "el campo que queremos» sino tan sólo advertir los variados desarrollos históricos que convergen en su articulación y sus posibles líneas actuales. El reconocimiento de un campo cultural es, por tanto, el reconocimiento de los esquemas de interpretaciones sobre su objeto (en este caso, el cine), la estructura de relaciones entre sus actores e instituciones y el grado de organicidad de sus prácticas.

Parece evidente, entonces, que el campo del cine no puede identificarse simplemente con alguna de las escenas en las que se desenvuelven sus actores o se desarrolla su actividad. El campo no es sólo el mercado cinematográfico, la escena académica, el espacio de la crítica, la producción o las instituciones estatales relativas a ella. Es el ámbito simbólico articulado en torno a las relaciones entre todas estas dimensiones, que configura nuestras percepciones y concepciones del cine y determina, asimismo, las acciones de cada uno de estos actores.

Esta noción de campo cultural es entendida aquí a la luz del concepto general de "campo" tal y como lo desarrolla el sociólogo Pierre Bourdieu (2002). El concepto, a su vez, ha sido enriquecido con el trabajo sobre prácticas, instituciones y formaciones culturales que propone Raymond Williams (1994). 
Se ha vuelto ya un lugar común afirmar que en las últimas décadas el cine en Chile ha tenido un desarrollo inusitado y saludable. ${ }^{2} \mathrm{Al}$ aumento explosivo de estrenos nacionales y salas de proyección se suma la proliferación de escuelas de cine, programas de estudio, festivales y encuentros sobre la materia. Al mismo tiempo, el papel del Estado en el fomento de cada una de las etapas del circuito cinematográfico (desde la subvención a la producción hasta el financiamiento de labores de difusión y restauración) parece consolidar lo que se volvería el propósito común de todo el campo del cine en Chile: la consolidación de una industria nacional. Promesa inveterada que expresa una aspiración de antigua data, que tiene sus hitos en los fallidos proyectos de Chilefilms durante las décadas que se extienden entre 1942 y 1973.

Como señala el crítico Iván Pinto (2008), este auge del cine en Chile parece ser no sólo de orden cuantitativo sino también el inicio de una tendencia hacia la estabilización y diversificación de este campo cultural. Parece oportuno y necesario, entonces, averiguar sobre las posibles correspondencias entre este desarrollo y la consolidación de un campo de estudios sobre el cine en Chile o, por el contrario, constatar las eventuales fisuras, diferencias, desniveles y desarticulaciones entre los espacios reflexivos e investigativos sobre cine y los demás espacios productivos, políticos y sociales del campo. Tal es el propósito de este texto, que dirige su mirada a tres ejes que permitirían obtener una visión panorámica, aunque aún provisoria, del campo de estudios: los discursos y modelos teóricos que se introducen, emplean y circulan en Chile; la conformación institucional del campo, especialmente la académica, pero también la relativa a la producción y al papel del Estado; y el conjunto de prácticas críticas y reflexivas respecto a las películas y al propio campo.

El panorama que se ofrece a continuación concentra su atención en los últimos cinco años de desarrollo del campo cultural, si bien es ineludible que, en ocasiones, extienda ciertas líneas interpretativas hacia el pasado, debido al irrecusable carácter histórico que comporta cualquier conformación sociocultural, de lo que el cine no es una excepción. Por otra parte, se trata tan sólo de una revisión parcial y exploratoria de las distintas densidades que podrían conformar el campo, pues no conocemos experiencias previas de reflexión sobre el campo mismo de estudios del cine ni están disponibles hoy todos los datos necesarios para formarnos una idea acabada sobre éste (lo cual, por sí mismo, constituye una limitación, aunque también un elemento para el análisis).

\section{DISCURSOS Y MODELOS TEÓRICOS EN LA CONFORMACIÓN DEL CAMPO DE ESTUDIOS}

En los últimos años un conjunto de textos han venido a ofrecer una colección de materiales, datos e interpretaciones inexistentes para el estudioso de hace dos décadas. Los trabajos contribuyen a un mayor conocimiento del cine producido en nuestro país en diversos ámbitos - el cine mudo, el cine político de los años 60 y 70, el cine de la transición, el documental- en la mayoría de los casos de un modo general. Los objetos de estudio suelen ser periodos históricos o ejes temáticos amplios. Existen textos que

De este entusiasmo dan cuenta Aliaga (2006), Villarroel (2005) y Estévez (2005), entre otros, así como innumerables artículos de prensa periódica que no tiene objeto referir en este lugar. 
contienen análisis sobre obras y realizadores específicos aunque, en varios casos, con un tratamiento más bien panorámico. ${ }^{3}$

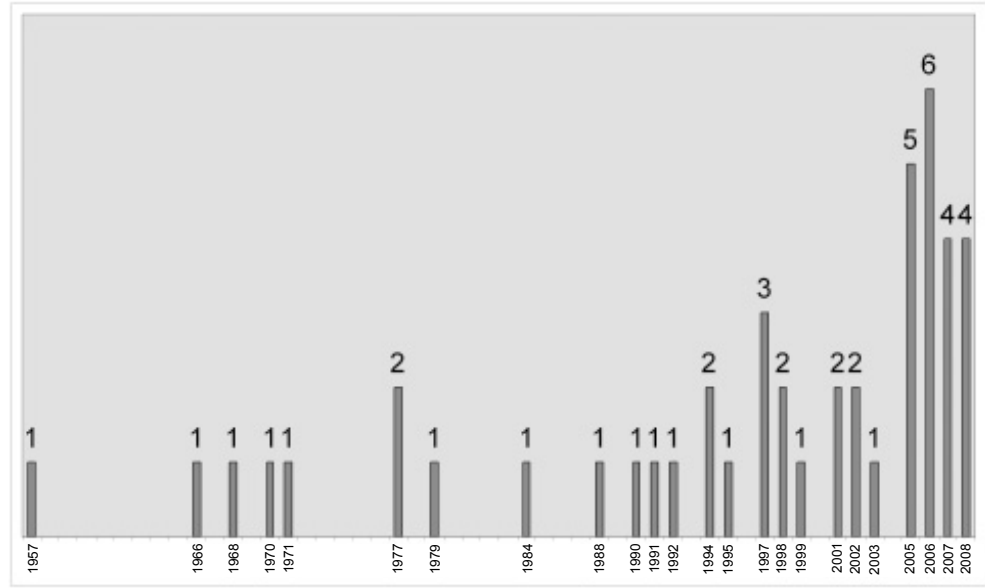

Figura 1. Libros sobre cine publicados en Chile por año, 1957-2008 (Fuente: elaboración propia).

Del catálogo bibliográfico chileno (que no se empina más allá de los cincuenta títulos), la mayoría de los escritos entre 1950 y 1990 corresponden a historias del cine. Estas historias inaugurarán lo que será una tendencia clara en la literatura: el énfasis en la narración de los acontecimientos del cine nacional. Hablamos de narración y no de historia pues estos libros no poseen un afán disciplinario, un especial manejo de datos ni la presentación de hipótesis.

Si se considera que la literatura general sobre cine no fue introducida en Chile sino hasta después de 1960, mediante la creación de diversos cine-clubes y la labor de los institutos culturales universitarios y bi-nacionales (el único texto conocido a mediados de los 50 es la Historia del cine de Georges Sadoul), es apreciable el magro panorama teórico. Obras como la Historia del cine chileno (1971) de Carlos Ossa Coó o el famoso Revisión del cine chileno (1979) de Alicia Vega aparecen como solitarios intentos no sólo de relatar el cine chileno sino también de tender líneas interpretativas sobre la inscripción del cine en el desarrollo de la industria nacional o el campo de la estética.

Al conjunto de historias y filmografías que se han producido desde 1990 hasta la fecha (periodo en el que la producción bibliográfica se cuadruplicó respecto de las cuatro décadas anteriores), apenas un puñado de textos ofrece estudios y evidencian algún tipo y nivel de reflexión teórica o metodológica. Entre ellos se encuentran los dos trabajos de Cavallo et al. Huérfanos y perdidos (1999) y Explotados y benditos (2007), los cuales proponen un análisis impresionista basado en la premisa de que el discurso de las películas refleja las condiciones sociales e históricas en que fueron éstas realizadas. Con una premisa similar, pero estudiando el discurso de los realizadores en lugar de las películas, se encuentran los textos de Antonella Estévez (2005) y Mónica Villarroel (2005).

3 Una revisión relativamente completa de la literatura sobre cine chileno puede revisarse en Salinas y Stange (2008). Este artículo, que comenta los libros publicados en la materia, deja aún pendiente una revisión crítica de las tesis y revistas especializadas. 


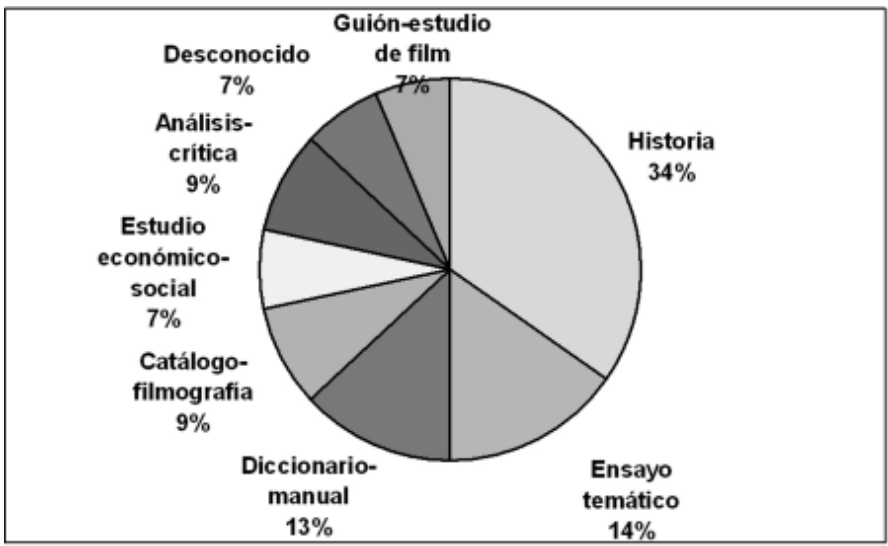

Figura 2. Libros publicados en Chile según temática, 1957-1973

(Fuente: elaboración propia).

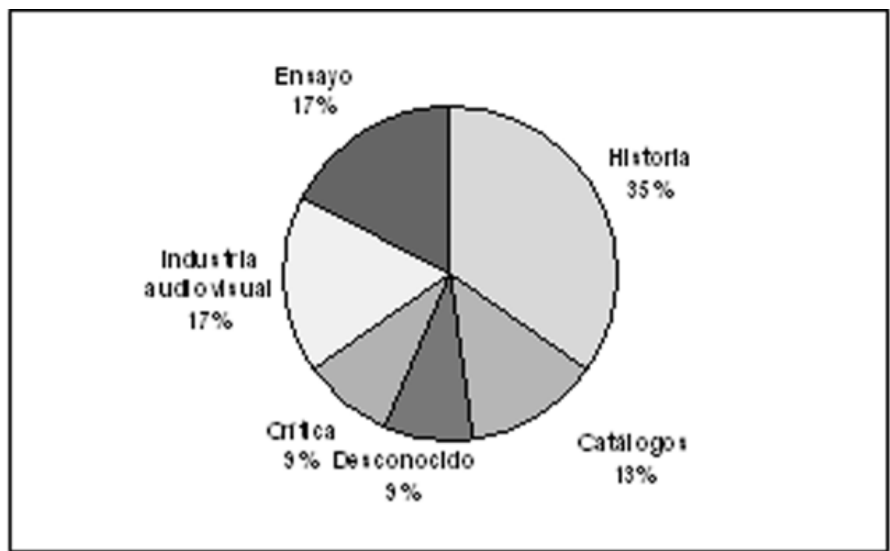

Figura 3. Proyectos financiados por el Fondo Audiovisual según temática, 2005-2008

(Fuente: elaboración propia).

Los trabajos que ofrecen mayor densidad en la formulación de una propuesta teórica para abordar el estudio de obras fílmicas son, seguramente, las monografías de Jorge Ruffinelli sobre Patricio Guzmán (2001) y Cristián Sánchez (2007), así como el libro Teorias del cine documental chileno 1957-1973 (2007) de Pablo Corro et al. En los tres casos hay un intento de mayor espesor reflexivo al abordar las obras desde la perspectiva de la construcción de una estética y un lenguaje audiovisual, sobrepasando el nivel de meros «reflejos» de la vida social.

Ninguno de estos trabajos, sin embargo, ha constituido escuela y se perciben más bien 
como intentos excepcionales dentro de un conjunto aún dominado por la narrativa histórica. Tampoco se ha dado pie al desarrollo de problemas estéticos o fílmicos específicos, ni se ha abierto el camino a perspectivas tangenciales, pero de gran interés teórico, como podrían ser, por ejemplo, la psicología del cine, la sociología del cine, la antropología visual u otras similares.

La misma dispersión temática y teórica se percibe en los proyectos realizados durante los últimos años con apoyo del Fondo Audiovisual. La investigación realizada hasta el año 2005 era a menudo fruto de la iniciativa individual de algunos estudiosos o de intereses marginales de departamentos o unidades académicas, las cuales escasamente alojaron proyectos de este tipo. Su ocurrencia, por tanto, fue esporádica, tal como lo atestigua la bibliografía reseñada. Desde 2005, el Consejo Nacional de la Cultura y las Artes (CNCA), a través de su Fondo Audiovisual, financia una línea de investigación y capacitación que ha subvencionado 25 proyectos de investigación. Todos estos proyectos responden a intereses, temáticas y perspectivas metodológicas y teóricas diferentes, en las que se considera desde la elaboración de un catálogo web hasta la propuesta de un modelo de marketing para cine chileno, pasando por trabajos sobre medios audiovisuales y redes neuronales. Nuevamente, la única tendencia sólida perceptible es la del relato de periodos, géneros o acontecimientos: la investigación histórica concentra nueve de los 25 proyectos $(36 \%)$.

En este contexto, ¿podría hablarse de corrientes o debates teóricos predominantes? ¿Existen énfasis y temas predilectos? Ciertamente hay «hitos» recurrentes en cuanto a temáticas y aproximaciones. Considerando, a modo de ejemplo, la bibliografía ya referida y los proyectos de investigación adjudicados por el CNCA, encontramos un total de 23 trabajos históricos, 11 ensayos o textos «teóricos», seis diccionarios, seis trabajos de crítica cinematográfica y siete estudios sobre mercado e industria audiovisual. Desde la perspectiva temática, encontramos cinco historias generales del cine chileno (escritas en su mayoría durante el periodo 1960-1980), cinco trabajos de historias periódicas (el cine mudo, el cine de los 60, el cine de la dictadura, el cine de la transición, etc.), seis trabajos sobre películas (desde El chacal de Nabueltoro hasta el guión de Taxi para tres) y otros tantos sobre realizadores (Patricio Guzmán, Cristián Sánchez, Raúl Ruiz, Alejandro Jodorowsky, etc.), cuatro trabajos sobre documental (escritos todos entre 2005 y 2008) y otros tres sobre ficción (escritos desde 1990 hasta la actualidad), entre otros. No obstante, estas coincidencias obliteran el hecho de que casi un tercio de la investigación no se incluye en estas clasificaciones, presentando las preocupaciones más variadas: desde la elaboración de catálogos regionales hasta monografías sobre videoclips o la relación del cine con la identidad nacional, la educación o la política.

Más importante aún: los trabajos que presentan coincidencias temáticas no manifiestan marcos epistemológicos definidos, un lugar interpretativo claro o un bagaje teórico y terminológico común. En otras palabras: puede haber seis investigaciones sobre un mismo periodo, por ejemplo, que no establecen diálogos entre sí, no tienen un acervo documental común, a veces ni siquiera un método de trabajo explícito o una nomenclatura suficientemente compartida. 
Tabla 1. Proyectos financiados por el Fondo Audiovisual, línea de investigación. 2005-2008 (fuente: CNCA).

\begin{tabular}{|c|c|c|c|}
\hline Año & Proyecto & Investigador responsable & Tipo de estudio \\
\hline \multirow{5}{*}{2005} & La magia en movimiento & Vivienne Barry Onfray & Artística \\
\hline & Teorías del cine documental en Chile 1957-1973 & Pablo Corro & Histórica \\
\hline & Catálogo web de documentales chilenos & ADOC & Histórica \\
\hline & Ampliación y profundización del proyecto U-matic & Germán Liñero & Histórica \\
\hline & Cines de Santiago, registro para el bicentenario & Paula Díaz Gallardo & Histórica \\
\hline \multirow{4}{*}{2006} & Aventura del cuerpo: el cine de Raúl Ruiz & Cristián Sánchez & Crítica \\
\hline & Historia del Cine Experimental en la U. de Chile & Claudio Salinas & Histórica \\
\hline & $\begin{array}{c}\text { Imágenes chilenas en el mundo: Catastro del acervo } \\
\text { audiovisual chileno en el exterior }\end{array}$ & $\begin{array}{l}\text { Centro Cultural Palacio La } \\
\text { Moneda }\end{array}$ & Histórica \\
\hline & La música en el cine chileno & Alejandra Polanco & Histórica \\
\hline \multirow{8}{*}{2007} & $\begin{array}{l}\text { Nuevos métodos de composición audiovisual basados } \\
\text { en lógica difusa, redes neuronales y percepción }\end{array}$ & Rodrigo Cádiz & Innovación tecnológica \\
\hline & $\begin{array}{c}\text { Educación, cine y memoria. Los usos del audiovisual } \\
\text { en procesos de educación para los Derechos } \\
\text { Humanos }\end{array}$ & Juan Pablo Donoso & Teórica \\
\hline & $\begin{array}{l}\text { Melodrama, subjetividad e historia. Ensayo sobre } \\
\text { la ficción cinematográfica y televisiva chilena en la } \\
\text { década del } 90\end{array}$ & $\begin{array}{l}\text { Pontificia Universidad Católica } \\
\text { de Chile }\end{array}$ & Teórica \\
\hline & $\begin{array}{l}\text { Pasado, presente y futuro de la realización audiovisual } \\
\text { en el sur de Chile }\end{array}$ & Rubén González & Histórica \\
\hline & $\begin{array}{c}\text { Investigación para la escritura de «La historia del } \\
\text { video en Chile» }\end{array}$ & Germán Liñero & Histórica \\
\hline & Catálogo del videoclip chileno: videoclip.cl & Carlos Moena & Histórica \\
\hline & $\begin{array}{l}\text { El proceso productivo en la industria audiovisual } \\
\text { nacional }\end{array}$ & Ignacio Aliaga & $\begin{array}{l}\text { Industria audiovisual y } \\
\text { mercados }\end{array}$ \\
\hline & El cine de Alejandro Jodorowski & Andrea Chignoli & Crítica \\
\hline \multirow{8}{*}{2008} & Joris Ivens en Chile: tres películas y su legado & Tiziana Panizza & Histórica \\
\hline & Observatorio de la ficción televisiva chilena & $\begin{array}{c}\begin{array}{c}\text { Pontificia Universidad Católica } \\
\text { de Chile }\end{array} \\
\end{array}$ & Histórica \\
\hline & $\begin{array}{l}\text { Técnicos cinematográficos chilenos, periodo entre } \\
\qquad 1960 \text { y } 1973\end{array}$ & Christian Morales & Histórica \\
\hline & $\begin{array}{l}\text { Crítica de la economía política del cine chileno } \\
\text { contemporáneo }\end{array}$ & Carlos Trejo & Teórica \\
\hline & Cine chileno, un espacio para el arte & Luis Machuca & Artística \\
\hline & $\begin{array}{l}\text { Miradas sobre el cine chileno, caso uno: El chacal de } \\
\text { Nahueltoro }\end{array}$ & Sergio Navarro & Artística \\
\hline & La imagen quebrada: ensayo audiovisual en Chile & Udo Jacobsen & $\begin{array}{l}\text { Nuevas tendencias } \\
\text { creativas }\end{array}$ \\
\hline & $\begin{array}{l}\text { Marketing de películas: un modelo de predicción de } \\
\text { difusión y ventas de cine en Chile }\end{array}$ & Emilia Paz González & Técnica \\
\hline
\end{tabular}

Al no constituir estos estudios un conjunto de modelos o tradiciones que sirvan de sustrato a la investigación subsecuente, pareciera el investigador estar obligado a reconstruir el ámbito y el objeto de su estudio cada vez, a volver a descubrir siempre los datos y las películas, a tratar toda fuente como si fuera de primera mano y todo problema como si se formulara por primera vez. El «adanismo» parece ser la condición teórica de los estudios de cine en Chile. 


\section{LAS INSTITUCIONES: ESTADO, MERCADO, ACADEMIA}

Parecería obvio pensar que el espacio de desarrollo natural de un campo de estudios sean las instituciones académicas. Sin embargo, la estructura actual de las unidades universitarias dedicadas al cine y al audiovisual está abocada principalmente a la formación de realizadores y profesionales. La orientación general de la oferta académica se encuentra dirigida al mercado, lo que supone, a su vez, que la escena universitaria ha fortalecido sus relaciones, dentro del campo cultural, con la producción y no con ámbitos reflexivos o ligados a la investigación.

Una prueba de esto es la organización de la oferta de programas de pregrado y postgrado en cine y comunicación audiovisual. Excluyendo los programas de formación técnica y aquellos referidos específicamente a la dirección y producción de TV, nos encontramos una amplia oferta de carreras de comunicación audiovisual y cine brindadas en más de una veintena de universidades e institutos profesionales. No todos los programas están vinculados a un grado académico o se asientan en un espectro "disciplinario" claro: estos conocimientos teóricos — provenientes principalmente de los ámbitos de la comunicación y el arte- apoyan formaciones marcadamente técnicas, orientadas a la realización y al desempeño de los egresados en el mercado audiovisual. Quizás las excepciones sean las escuelas del Instituto Arcos y de la Universidad de Chile, paradójicamente, una de las más antiguas y otra de las más recientes en este ámbito formativo.

Tabla 2. Programas de pre y postgrado en cine y audiovisual vigentes en Chile, 2009 (Fuente: Mineduc).

\begin{tabular}{|c|c|c|}
\hline Tipos de Programa & $\begin{array}{c}\text { Cantidad de Instituciones que lo } \\
\text { imparten }\end{array}$ & $\begin{array}{c}\text { Matrícula de ingreso del } \\
\text { año } 2009 \\
\end{array}$ \\
\hline \multicolumn{3}{|c|}{ I. PREGRADO } \\
\hline Dirección y producción de TV & 1 instituto profesional & 35 \\
\hline Comunicación audiovisual & $\begin{array}{c}4 \text { institutos profesionales } \\
3 \text { universidades }\end{array}$ & C. 875 \\
\hline Cine & $\begin{array}{c}1 \text { instituto profesional } \\
9 \text { universidades }\end{array}$ & c. 275 \\
\hline Técnico audiovisual & 2 institutos profesionales & 240 \\
\hline Dirección audiovisual & 1 universidad & $s / i$ \\
\hline \multicolumn{3}{|c|}{ II. POSTGRADO } \\
\hline Diplomado en realización & 2 universidades & c. 40 \\
\hline Postítulo profesional & 2 universidades & 36 \\
\hline Diplomado de estudio o especialización & 3 universidades & c. 50 \\
\hline
\end{tabular}

En cualquier caso, los programas más asentados, reconocidos y de mayor matrícula se instalan en una perspectiva casi exclusivamente profesionalizante. ¿Cuál es entonces el lugar de la reflexión en el proceso formativo? ¿Qué capacidad tiene este mercado para producir, además de realizadores, otros perfiles necesarios al interior del campo cultural del cine? Si bien parece natural que casi la mitad de la planta docente esté conformada por realizadores — pues se trata de programas orientados a la realización- es notoria 
la manera en que otros ámbitos del campo cultural, como la crítica, la investigación, la gestión del mercado y la industria, quedan en manos de profesionales cuya experticia primera no es el cine: periodistas, sociólogos, incluso abogados e ingenieros.

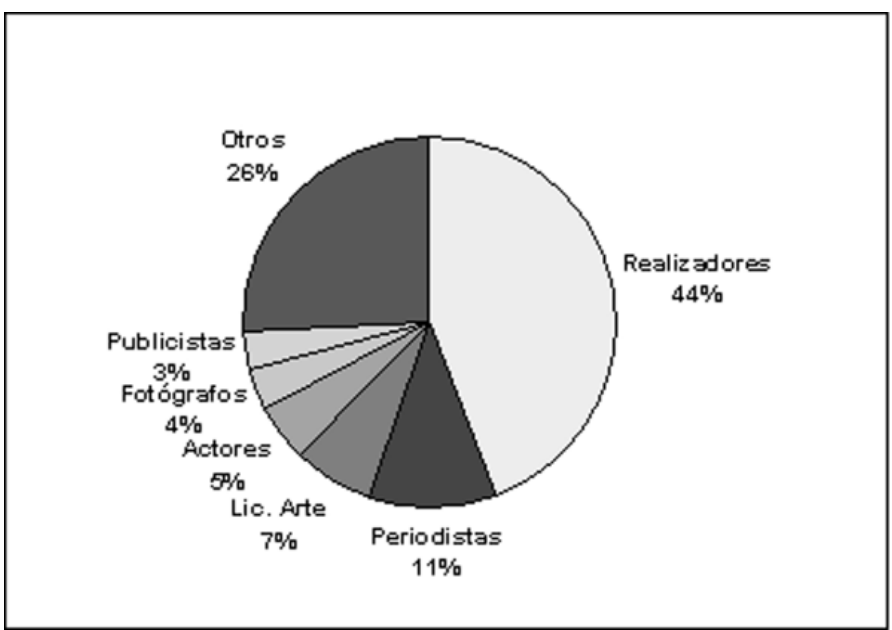

Figura 4. Procedencia de los cuerpos docentes de los programas de pre y postgrado (Fuente: elaboración propia sobre la información «incompleta» de los planteles en sus sitios web). Otros: Sonidistas, literatos, sociólogos, filósofos, historiadores, músicos, diseñadores, arquitectos, ingenieros, psicólogos, antropólogos, abogados.

Lo anterior, que podría suponer positivamente una apertura interdisciplinaria, parece ser más bien la evidencia de un ajuste no alcanzado en el diseño de los planes de estudios. El acento en la realización no impide que los planes reciban contribuciones desde los campos del arte, la comunicación y la historia, principalmente, no obstante, por ejemplo, escasea la formación de los futuros egresados en ciencias sociales o humanidades. Al mismo tiempo, esto no supone una definición «disciplinaria» del cine, pues el grado de especificidad evidenciado es más bien producto de la orientación profesional que de una definición epistémica o cultural sobre lo «propio» del cine.

También los postgrados se orientan a la producción, con una oferta conformada principalmente por diplomados de especialización y realización, salvo algunas pocas excepciones. Es interesante notar que las universidades que ofrecen postgrados no son las mismas que ofrecen carreras de pregrado, lo cual indica que por sobre una concepción de la formación continua - aun de carácter profesional-, prima un «sentido de la oportunidad» por parte de estas instituciones al momento de diseñar y abrir estos programas.

Tal correspondencia entre el espacio académico y el mercado pareciera incluso ir en desmedro de las posibilidades de consolidación del campo de estudios en las universidades. La proliferación de programas y mallas curriculares no ha significado necesariamente la aparición de publicaciones especializadas, centros de estudios o cuerpos de investigadores. Las universidades no estarían jugando un rol estratégico para el cual estarían posibilitadas, como constituirse en el espacio de vinculación entre producción e 
investigación. Más bien se habrían alineado casi completamente con los requerimientos del mercado, optando por un único modelo de desarrollo.

Debiera considerarse que la presencia del cine en los planteles académicos ha sido históricamente marginal y no ha respondido, en la mayoría de los casos, a ninguna política universitaria respectiva. Las primeras incursiones universitarias relativas al cine surgieron con el apoyo a centros de producción marginales o experimentales. Luego, las primeras carreras abiertas en los años 60 y 70 expresaban intereses académicos difusos y entremezclados con potentes discursos de transformación política y cultural, amén de los proyectos de industrialización del audiovisual propios de la época. Esto explicaría que las concepciones del cine en Chile no aparezcan vinculadas a proyectos o tradiciones académicos, sino que se perciban y articulen marcadas por las contingencias y la contemporaneidad de las preocupaciones de cada época particular.

Del mismo modo que la academia, también el Estado ha asumido como horizonte directivo el fomento a una arista particular de este campo cultural: la producción. Históricamente, los propósitos de la política estatal en relación al cine se han orientado a la consolidación de un mercado y una industria audiovisuales. Ésta fue la experiencia de Chile Films entre las décadas de 1940-1970 y es también la prerrogativa de las políticas estatales en la actualidad, con las particularidades evidentes en cada caso. Podría incluso señalarse que fuera de las subvenciones a la producción y los estímulos al comercio no hay, de parte del Estado, ninguna política «cultural» hacia el cine.

Tabla 3. Evolución comparada de los montos de fomento a la producción y la investigación en cine por parte del Estado, 2006-2009 (Fuente: elaboración propia sobre datos del CNCA).

\begin{tabular}{|c|c|c|c|c|}
\hline Año & 2006 & 2007 & 2008 & 2009 \\
\hline $\begin{array}{c}\text { Total de } \\
\text { aportes del } \\
\text { Estado }\end{array}$ & $1.430 .800 .000 .-$ & $2.478 .800 .000 .-$ & $1.198 .000 .000 .-$ & $1.642 .500 .000 .-$ \\
\hline $\begin{array}{c}\text { Fondos para } \\
\text { investigación }\end{array}$ & $69.000 .000 .-$ & $96.670 .000 .-$ & $79.000 .000 .-$ & $60.000 .000 .-$ \\
\hline $\begin{array}{c}\text { \% de } \\
\text { fondos para } \\
\text { investigación } \\
\text { respecto del } \\
\text { total }\end{array}$ & $4,8 \%$ & $3,9 \%$ & $6,6 \%$ & $3,65 \%$ \\
\hline
\end{tabular}

Las subvenciones a la investigación, en cambio, si bien suponen un apoyo sustantivo a un área que no recibía ninguna atención institucional, son marginales en relación al aporte global del Estado a la «industria audiovisual». Durante los cuatro años de existencia de la línea de investigación y capacitación del Fondo Audiovisual, ésta ha percibido apenas el $4,7 \%$ promedio del total de los fondos, con montos absolutos decrecientes desde el segundo año de aplicación (2006) hasta la fecha. 
La política estatal tampoco aplica medidas de intervención correctiva al mercado audiovisual ni medidas cautelares de la producción nacional: limita su acción al propiciamiento de producciones competitivas y comercialmente exitosas (en lo posible) sin dictaminar, al menos explícitamente, pautas temáticas o ideológicas particulares (reconociendo que la alineación con el mercado es ya, de por sí, una acción altamente ideologizada). En efecto, incluso otros organismos estatales o con participación estatal como CORFO, el Consejo Nacional de Televisión o Ibermedia, no intervienen en el campo cultural de otra forma que no sea subvencionando algunas o todas las etapas de la producción audiovisual (desde la elaboración del guión hasta la distribución en salas).

En este sentido, el papel del Estado, en relación a la investigación, se limita a la generación necesaria, pero muchas veces dispersa e incompleta, de estudios estadísticos y técnicos sobre la composición de la industria, los mercados o las audiencias. Estos estudios no tienen la difusión debida ni dialogan críticamente con el resto de la literatura o el mercado mismo.

\section{LAS PRÁCTICAS REFLEXIVAS Y CRÍTICAS}

La conformación de un campo de estudios sobre cine al interior del campo cultural del cine mismo aparece como una empresa fragmentaria, dispersa e incompleta. La escasa literatura producida por estudiosos e investigadores no ha logrado organizar perspectivas de interpretación consolidadas ni núcleos comprensivos, modélicos y reconocibles como fundamentales para la organización y articulación de los discursos críticos y teóricos. En su conjunto, los escasos estudios sobre cine realizados en el país no ofrecen una visión consistente que permita describir y reconocer este campo cultural, que sirva de fundamento a su producción y provea de marcos interpretativos para el conocimiento de sus cambios, transformaciones e innovaciones.

Por otro lado, los ámbitos de la producción, el Estado y la formación académica, que también participan de este campo cultural, no han logrado - o no se han propuestoarticular los espacios y escenas de aparición que permitan la emergencia y circulación de los discursos críticos y teóricos. La orientación general de buena parte del campo cultural ha copado los espacios de circulación y los recursos movilizados, tornando «irrelevantes» otras esferas de producción simbólica. Por otra parte, la desarticulación institucional que subyace a esta alineación general con el mercado ha restado el necesario espacio de legitimación para los estudios: la academia y las políticas estatales no han requerido el concurso del campo de estudios para el desarrollo de la «industria audiovisual» ni han admitido lógicas de organización del campo cultural distintas de la que hoy es hegemónica y que tiene como único principio organizador el mercado.

Estas constataciones obligan a pensar cuál sería el lugar que les corresponde a las prácticas críticas y reflexivas. Marginadas de las escenas institucionales y desvinculadas de las lógicas productivas, las prácticas críticas aparecerían no sólo como innecesarias para el campo cultural del cine, sino que también como improcedentes e, incluso, impertinentes. En este contexto, una somera revisión, por cierto parcial, de la conformación histórica de estas prácticas discursivas pondría en evidencia al menos dos cuestiones, 
primero, el hecho de que estas prácticas no se organizan en torno a los saberes específicos del cine ni en torno a los modelos teóricos que, como hemos visto, son difusos e inorgánicos; segundo, y a causa de lo anterior, que el conjunto de las prácticas críticas y reflexivas suele apoyarse en los saberes teóricos de otros campos, en los cuales se asienta y legitima a riesgo de producir malas «traducciones» teóricas entre un campo y otro y, a la vez, perpetuar la inarticulación del campo de estudios debido a la heterogeneidad de estos «préstamos» teóricos.

Lo anterior es evidente en la revisión de las bibliografías de artículos, tesis, estudios y cursos de cine. En el caso de éstos últimos, y excluyendo desde un comienzo la enorme cantidad de literatura técnica (propia de una formación orientada a la profesionalización) las bibliografías teóricas se organizan en torno a núcleos que van desde la semiología a la crítica cultural, pasando por teorías de la comunicación, textos de arte y estética y ensayos sobre cultura en general. Quizás la mayor especificidad se encuentre, una vez más, en los textos de historia del cine, que conformarían el corpus más estable dentro de los currículos. Otros textos sobre lenguaje audiovisual, teorías del montaje, etc., por su carácter manualístico estarían igualmente siguiendo una orientación profesional.

Un caso similar se observaría en las bibliografías referidas en los artículos de las escasas revistas de cine y crítica especializada. La abundancia de referencias a lecturas provenientes de la crítica cultural, la filosofía del lenguaje, el postestructuralismo o las artes postmodernistas podría significar un mayor interés por interpretar el cine a partir de matrices culturalistas que una necesidad de suplir la ausencia de modelos discursivos teóricos específicos, lo cual tiene, nuevamente, como problema potencial, la dispersión de los discursos. Lo anterior no significa en ningún caso denostar estos acercamientos, pero sí pone en evidencia la tensión que supone el que tales discursos no tengan una contraparte, generada al interior del propio campo, con la cual establecer diálogos y relaciones.

Otro aspecto que evidencia la desarticulación de las prácticas al interior del campo del cine es la procedencia y formación de los autores, docentes e investigadores que intervienen en él. Nuevamente, no se aboga aquí por un dogmatismo disciplinario sino que se pretende advertir que aquello que pudiera leerse bajo claves cooperativas o multidisciplinarias puede entenderse también como simple carencia en la medida en que es apreciable la ausencia de relaciones o diálogos entre estos elementos.

Naturalmente, debido al hecho de que muchos de los programas de estudios sobre cine y audiovisual son recientes (también a causa de la ya mencionada posición marginal del cine al interior de las instituciones de educación superior), un número importante de investigadores y autores presenta una formación muy heterogénea, informal si no autodidacta, conseguida mediante esfuerzos e iniciativas personales antes que por adscripciones institucionales formales. Es notorio que, del total de autores de literatura sobre cine en Chile, apenas un $22 \%$ tenga estudios formales de cine. El resto de los autores está constituido por periodistas (24\%), distintos representantes de disciplinas artísticas y de ciencias sociales (32\%) y un no despreciable número de autores cuya formación se desconoce (19\%). Del mismo modo, un $11 \%$ de los cuerpos docentes está constituido por periodistas, un $7 \%$ por licenciados en arte, un $5 \%$ por actores o actrices y, en porcentajes variables, se encuentran también publicistas, sociólogos, filósofos, arquitectos, ingenieros civiles y comerciales, psicólogos y abogados.

Estos datos no invalidan los juicios planteados anteriormente en el sentido de que la orientación profesionalizante de las carreras motiva una concentración de las plantas 
docentes en la figura del realizador o comunicador audiovisual. Éstos alcanzan al 44,2\% de las plantas académicas, repartiéndose el resto de las plazas entre la variedad de profesionales ya señalada.

\section{PREGUNTAS FINALES}

¿Qué propósito puede tener la constitución de un campo de estudios al interior de un circuito cultural que parece haber optado ya por concentrar sus esfuerzos solamente en la producción? ¿Qué papel podría cumplir este campo de estudios, en el caso de consolidarse efectivamente, en la modificación de nuestras percepciones sobre la realización fílmica, en la movilización de una serie de discursos críticos hoy ausentes o en la definición de orientaciones respecto a la relación del cine con el resto del campo cultural y social?

Para intentar una respuesta satisfactoria a estas tareas se impone a futuro un trabajo mayor de sistematización y recopilación de datos hoy incompletos y parciales, una caracterización histórica de los procesos de formación de los planes de estudios, bibliografías y cuerpos académicos e investigadores; una revisión crítica de los posibles diálogos, juicios y articulaciones teóricas entre los textos. El reclamo por la conformación del campo de estudios parece ser, curiosamente, la única forma de establecer una claridad sobre su lugar, su objeto y su propósito.

\section{REFERENCIAS}

Aliaga, Ignacio. (2006). Cine de Chile 1990-2005. La pequeña historia de una imagen obstinada. La cultura durante el periodo de transición a la democracia 1990-2005. Valparaíso: Consejo Nacional de la Cultura y las Artes.

Bourdieu, Pierre. (2002). Campo de poder, campo intelectual. Itinerario de un concepto. Buenos Aires: Montressor.

Cavallo, Ascanio y Carolina Díaz. (2007). Explotados y benditos. Mito y desmitificación del cine chileno de los 60. Santiago: Uqbar.

Cavallo, Ascanio, et al. (1999). Huérfanos y perdidos. El cine chileno de la transición 1990-1999. Santiago: Grijalbo.

Consejo de Educación Superior. (2009). Obtenido desde <http://www.educacionsuperior.cl>.

Consejo del Arte y la Industria Audiovisual. (2009). Obtenido desde <http://www.chileaudiovisual.cl>.

Consejo Nacional de la Cultura y las Artes. (2009). Obtenido desde <http://www.cnca. $\mathrm{cl}>$.

Corro, Pablo, et al. (2007). Teorías del cine documental chileno 1957-1973. Santiago: Pontificia Universidad Católica de Chile.

Estévez, Antonella. (2005). Luz, Cámara, Transición. El rollo del cine chileno de 1993 a 2003. Santiago: Radio Universidad de Chile. 
Flores, Carlos. (2008). Excéntricos y astutos. Revista La Fuga. Obtenido desde <www. lafuga.cl>.

Muñoz, Richard. (2008). A propósito de la industria cultural. ¿El estado de las cosas o las cosas del Estado? Revista La Fuga. Obtenido desde <www.lafuga.cl>.

Ossa Coó, Carlos. (1971). Historia del cine chileno. Santiago: Quimantú.

Pinto, Iván. (2008). Mercado y contingencia. Revista La Fuga. Obtenido desde sitio $<$ www.lafuga.cl $>$.

Revista Fuera de Campo. (2009). Obtenido desde <http://www.fueradecampo.cl>.

Revista Mabuse. (2009). Obtenido desde <http://www.mabuse.cl>.

Ruffinelli, Jorge. (2001). Patricio Guzmán. Madrid: Cátedra.

-. (2007). El cine nómada de Cristián Sánchez. Santiago: Uqbar.

Salinas, Claudio y Hans Stange. (2008). La incipiente literatura sobre cine chileno: obra en construcción. Revista La Fuga. Obtenido desde <www.lafuga.cl>.

Vega, Alicia. (1979). Re-visión del cine chileno. Santiago: Aconcagua-CENECA.

Villarroel, Mónica. (2005). La voz de los cineastas. Cine e identidad en el umbral del milenio. Santiago: Cuarto Propio.

Williams, Raymond. (1994). Sociología de la cultura. Barcelona: Paidós.

Ya no basta con filmar. Informe de la industria audiovisual en Chile. (2008). Santiago: Pontificia Universidad Católica de Chile. 